\title{
Impact of treatment dose on patient outcomes after Interdisciplinary Multimodal Pain Therapy
}

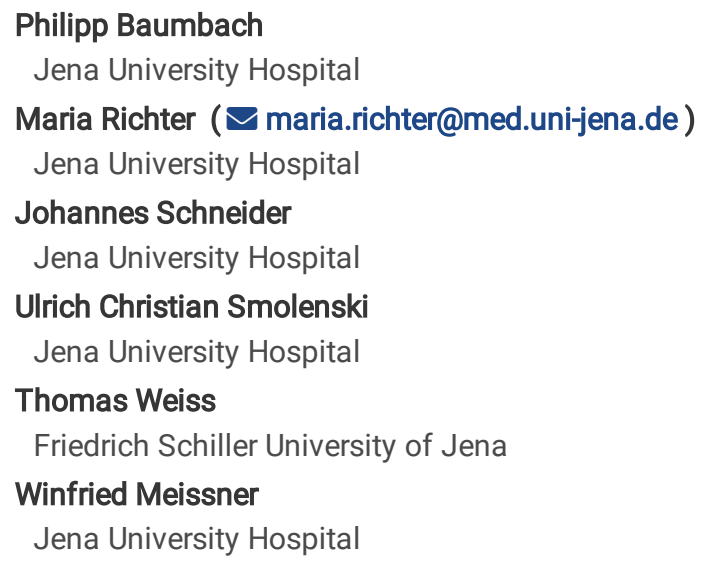

\section{Research Article}

Keywords: Interdisciplinary Multimodal Pain Therapy, Chronic pain, Therapy Dose

Posted Date: May 19th, 2021

DOI: https://doi.org/10.21203/rs.3.rs-514829/v1

License: (c) (i) This work is licensed under a Creative Commons Attribution 4.0 International License. Read Full License 


\section{Abstract}

Objectives The impact of duration and intensity on outcomes after Interdisciplinary Multimodal Pain Therapy (IMPT) is poorly researched. The aim of this study was to compare the effects of low dose (LD, avg. 25 hours) and high dose IMPT (HD, avg. 110 hours).

Methods Patients completed pain-related questionnaires at the beginning (T1), at the end of therapy (T2) and at 3-month follow-up (T3) and were matched according to age, sex, presence of back-pain and pain-related disability at $\mathrm{T} 1$, resulting in 32 patients per group. Primary endpoint was the difference in pain-related disability and average pain intensity at T3 between both groups. In addition, early treatment effects and group differences at $\mathrm{T} 2$ were analyzed.

Results Both groups showed significant improvements in pain-related disability and average pain intensity between T1 and T2. These positive effects persisted in the HD group until the 3-month follow-up, whereas outcomes in the LD group patients deteriorated and were significantly poorer compared to $\mathrm{HD}$ at T3.

Discussion Within a widely comparable therapeutic setting, high-dose IMPT was associated with longer lasting improvements compared to lowdose IMPT in chronic pain patients, indicating that the "dose" of therapy is a relevant factor for clinical outcomes and should be further investigated.

\section{Introduction}

Chronic pain is a highly prevalent condition with considerable individual, social and societal consequences ${ }^{1,2}$. Although systematic research is challenged by heterogeneous therapeutic and structural factors (e.g. setting, type and duration of treatment elements, composition of team members), and different outcome measures, the treatment- and cost-effectiveness of Interdisciplinary multimodal pain therapy (IMPT) was reported repeatedly and led to first-line recommendation of IMPT for chronic pain patients ${ }^{3-13}$.

Several reviews discussed the association between dose aspects and effectiveness of IMPT programs for patients with chronic low back pain. The term dose mainly refers to therapy duration, i.e. total number of contact hours. The term dosage refers to intensity of a treatment within a certain time frame (e.g. 3 hours per week). One review concluded that programs with $>100$ hours of treatment were more effective compared to programs with $<30$ hours of treatment or compared to unimodal treatments ${ }^{14}$. In contrast, another review found that intensity of treatment, as aspect of dosage, had no substantial effect on long-term-effectiveness, although most of the 10 studies included did not compare different IMPT programs directly ${ }^{15}$. Additionally, some of the included studies evaluated treatment programs (e.g. "fitness training", ${ }^{16}$ ) with lack of an interdisciplinary therapeutic concept (e.g. ${ }^{17}$ ) or solely compared different low-dosed IMPT programs (e.g. ${ }^{18}$ ). A review including 27 studies reported a superior long-term effectiveness for (more intense) inpatient compared to (less intense) outpatient treatments, but a direct association between the number of treatment hours and effectiveness could not be stated ${ }^{19}$. Another review systematically analyzed doseeffects of pain rehabilitation programs controlling for possible confounders and found a positive effect of duration in weeks on therapy outcome. A clear effect of duration could not be stated because of the high association between duration and number of disciplines or contents involved ${ }^{20}$.

Likewise, the most recent reviews failed to find overall dose effects, due to the complex methodological difficulties, e.g. large differences regarding clinical settings, contact hours, therapeutic contents, proportion of contents, implementation of interdisciplinary team consultations and number of disciplines involved ${ }^{21}$.

Only few studies directly compared low and high dose interventions. Recently, it was shown that effectiveness might also depend on patients' prognosis, i.e. patients with good prognosis benefitted equally from light and extensive IMPT, while patients with poor prognosis (associated with higher physical and psychosocial malfunctions) showed higher benefits from extensive IMPT ${ }^{22}$. From that point of view, it is close to infer that patients with higher symptom severity and higher pain-related disability benefit more from high intense IMPT programs of longer duration. So far, this aspect was not explicitly considered in previous studies or reviews.

Although it seems difficult to vary dose of IMPT without affecting other variables, e.g. clinical setting, team features and patients characteristics such as severity of symptoms, a direct comparison of different treatment doses is exceptionally valuable for further research on IMPT outcomes and cost-efficacy aspects ${ }^{20}$.

We hypothesize that patients characterized by chronic pain conditions and high pain-related disability benefit more from high dose (HD) compared to low dose (LD) IMPT in the 3-month follow-up.

\section{Materials And Methods}


Patients and setting. All patients received IMPT at Jena University Hospital, based on cognitive-behavioral, educational, and physically activating approach with the overall aim of functional restoration. The treatment content was similar in both groups and comprised optimization of medical treatment, physical activation, occupational therapy, relaxation therapy, and psychological pain management. The proportion of those therapeutic elements was comparable between groups. The treatment team consisted of pain physicians, psychologists/psychotherapists, physiotherapists, occupational therapists, and pain nurses. Patients were referred to IMPT by several hospital departments or GPs. The setting was mainly group therapy (approximately $80 \%$ of contact hours in both groups) and a minor proportion of individual therapy sessions.

The LD-IMPT took place individually or in groups up to 4 patients between January 2009 and November 2010 in an inpatient setting. An interdisciplinary assessment took place on the first in-patient-day prior to treatment. Therapeutic interventions lasted approximately 25 hours on 7 treatment days ( 1 week), and interdisciplinary team sessions were held for $2-3$ hours per therapy group. Patients received the follow-up questionnaire per mail (postal service) 12 weeks after therapy.

The HD-IMPT was performed in groups of 5 to 9 patients between June 2013 and March 2017 in a day-clinic setting. An interdisciplinary assessment was conducted on one full day approximately 2-3 months prior to treatment. Therapeutic interventions lasted approximately 110 hours on 20 treatment days (4 weeks) and 2 refresher days. The interdisciplinary team sessions took 14-15 hours per therapy group.

Beside the mentioned differences in treatment duration and group size, there was a high degree of overlapping therapeutic and environmental conditions due to the fact that almost the same disciplines, therapeutic interventions and contents and in part even the same staff members were involved. All methods and experimental protocols were carried out in accordance with relevant guidelines and regulations. Ethical approval was obtained from the local ethics committee of the Jena University Hospital. Informed consent was obtained from all subjects.

Questionnaires. All patients completed the German Pain Questionnaire (DSF, ${ }^{23}$ ) at the first day (T1), the last day of treatment (T2), and at a 3month follow-up (T3).

Outcome scales. We used the German version of the Chronic Pain Grade Questionnaire ${ }^{24,25}$ as part of the DSF 23 . Average and maximum pain intensity during the last 7 days was assessed on an 11-point numeric rating scale (NRS, $0=$ no pain to $10=$ worst imaginable pain). Pain-related disability was assessed with 3 items asking how much pain interfered with daily living, work performance, and recreational/social/family activities within the last 7 days (11-point NRS, $0=$ no interference to $10=$ most severe interference). The pain-related disability scale was defined as mean of the three items ${ }^{24,25}$.

Matching procedure. To achieve comparability in baseline data and to reduce differences in sample size, LD- and HD-patients were matched 1:1 (see electronic Supplement 1). The DSF was revised in 2011 and the Hospital Anxiety and Depression Scale (HADS) ${ }^{26,27}$ was replaced with the German Depression Anxiety and Stress Scale (DASS) ${ }^{28,29}$. To ensure consistent scales within the LD- (HADS) and HD-group (DASS) for baseline characterization, HD-patients who completed the HADS $(n=28)$ were excluded from the matching procedure.

First, we exactly matched according to sex and the presence of chronic back pain (yes vs. no). Second, patients were matched according to age and the pain-related disability score (see below) at T1 within the respective subclasses (females and back pain: no | females and back pain: yes | males and back pain: no | males and back pain: yes) using "nearest neighbor" matching. Matching was performed using the 'matchlt' package 30 in R ( ${ }^{31}$, Version 3.5.1, R Foundation for Statistical Computing, Vienna, Austria).

Statistical analyses. The primary endpoint was the difference of the pain disability scale and average pain intensity at T3 (3-month follow-up) between both groups. Secondary outcomes comprised the initial therapy effects in both groups (T1 vs. T2) and group differences in painrelated disability scale and average pain intensity at $\mathrm{T} 2$.

Dichotomous data is presented in absolute and relative frequencies. Continuous data is presented in median and first/third quartiles $\left(\mathrm{Q}_{1} / 3\right)$. Because of the matching procedure, group differences were analyzed via McNemar tests for dichotomous and Wilcoxon signed-rank tests for continuous data. Differences between time-points were analyzed in the same way. Effect sizes are reported as $r$ coefficients and can be interpreted as follows: large $\geq 0.5$, medium $\geq 0.3$, small effect $\geq 0.1^{32}$.

We applied a significance level of $5 \%$ and we report two-sided p-values for comparisons between groups and time points. For the analyses, we used R (Version 3.5.1, R Foundation for Statistical Computing, Vienna, Austria) ${ }^{31}$ and SPSS (Version 22.0, IBM Corp., Armonk, N.Y., USA).

\section{Results}

The final study sample comprised 32 LD- and 32 HD-patients (for details see Fig. 1 and electronic Supplements 1 to 3 ). After matching, there were no significant differences in baseline demographic, treatment-related (see Table 1) and pain-related data (see Table 2) between both 
Table 1

Demographic and treatment-related patient data for both groups. Median and first/third quartile $\left(\mathrm{Q}_{1} / \mathrm{Q}_{3}\right)$ are displayed for continuous data. Dichotomous data is displayed in number (n) and percent (\%). P-values of the group comparisons ate the beginning of the interdisciplinary multimodal pain therapy (IMPT) are displayed in the last column (p).

\begin{tabular}{|c|c|c|c|c|c|c|c|c|}
\hline & & \multicolumn{3}{|c|}{$\begin{array}{l}\text { low-dose IMPT } \\
(\mathrm{n}=32)\end{array}$} & \multicolumn{4}{|c|}{$\begin{array}{l}\text { high-dose IMPT: matched } \\
(\mathrm{n}=32)\end{array}$} \\
\hline & & median & $\mathbf{Q}_{1}$ & $\mathbf{Q}_{3}$ & median & $Q_{1}$ & $\mathbf{Q}_{3}$ & $\mathbf{p}^{1}$ \\
\hline age & (years) & 55.0 & 47.8 & 69.8 & 54.5 & 50.3 & 63.3 & .927 \\
\hline \multirow[t]{2}{*}{ follow-up } & (days after T2) & 79.5 & 71.0 & 95.3 & 84.5 & 73.3 & 96.0 & .614 \\
\hline & & $\mathbf{n}$ & $\%$ & & $\mathbf{n}$ & $\%$ & & $\mathbf{p}^{2}$ \\
\hline sex & (female) & 15 & 46.9 & & 15 & 46.9 & & .999 \\
\hline chronic pain duration & ( $\geq$ 5years) & 19 & 59.4 & & 19 & 59.4 & & .999 \\
\hline chronic back pain & (yes) & 23 & 71.9 & & 23 & 71.9 & & .999 \\
\hline symptoms of anxiety & $(\text { above cut-off })^{3}$ & 14 & 43.8 & & 14 & 43.8 & & .999 \\
\hline symptoms of depression & $(\text { above cut-off) })^{4}$ & 12 & 37.5 & & 11 & 34.4 & & .999 \\
\hline \multicolumn{9}{|c|}{${ }^{1}$ p-values result from Wilcoxon signed-rank tests } \\
\hline \multicolumn{9}{|c|}{${ }^{2} \mathrm{p}$-values result from McNemar tests } \\
\hline \multicolumn{9}{|c|}{$\begin{array}{l}\left.{ }^{3} \text { low-dose patients: sum scores of the Hospital Anxiety Depression Scale (HADS, }{ }^{26,27,39}\right) \text { : Anxiety } \geq 11 \text { or } \\
\text { high-dose patients: sum score of the Depression, Anxiety and Stress Scale (DASS, }{ }^{28,29,40} \text { ): Anxiety } \geq 6\end{array}$} \\
\hline \multicolumn{9}{|c|}{$\begin{array}{l}{ }^{4} \text { low-dose patients: sum scores of the Hospital Anxiety Depression Scale (HADS, }{ }^{26,27,39} \text { ): Depression } \geq 11 \text { or } \\
\left.\text { high-dose patients: sum score of the Depression, Anxiety and Stress Scale (DASS, }{ }^{28,29,40}\right) \text { : Depression } \geq 10\end{array}$} \\
\hline
\end{tabular}

Table 2

Median, first and third quartile $\left(Q_{1} / Q_{3}\right)$ of the pain-related questionnaire data for both patient groups. Bonferroni-Holm corrected $p$-values ( $\left.p_{T 1}\right)$ from Wilcoxon signed-rank tests comparing values at the start (T1) vs. end (T2) of the interdisciplinary multimodal pain therapy (IMPT) and T1 vs. follow-up (T3) within both groups are displayed. The corresponding effect sizes are listed under column $\left|\mathrm{r}_{\mathrm{T} 1}\right|$. In addition, two-sided $\mathrm{p}$-values from Wilcoxon signed-rank tests comparing both groups $\left(\mathrm{p}_{\text {group }}\right)$ and corresponding effect sizes $\left(\mid \mathrm{r}_{\text {group }}\right)$ are displayed.

\begin{tabular}{|c|c|c|c|c|c|c|c|c|c|c|c|c|c|c|}
\hline & & & $\begin{array}{l}\text { low-dose } \\
(n=32)\end{array}$ & IMPT & & & & $\begin{array}{l}\text { high-dos } \\
(n=32)\end{array}$ & IMP & mat & & & & \\
\hline scale/item & [units] & time & median & $\mathrm{Q}_{1}$ & $\mathrm{Q}_{3}$ & $\mathbf{p}_{\mathrm{T} 1}$ & $\left|\mathbf{r}_{\mathrm{T} 1}\right|$ & median & $\mathrm{Q}_{1}$ & $\mathrm{Q}_{3}$ & $\mathbf{P}_{\mathrm{T} 1}$ & $\left|\mathbf{r}_{\mathrm{T} 1}\right|$ & $\mathbf{P}_{\text {group }}$ & $\left|\mathbf{r}_{\text {group }}\right|$ \\
\hline \multirow[t]{3}{*}{$\begin{array}{l}\text { disability: } \\
\text { scale }\end{array}$} & $\begin{array}{l}{[0-} \\
10]\end{array}$ & $\mathrm{T} 1$ & 5.3 & 3.9 & 6.9 & & & 5.0 & 4.0 & 6.2 & & & .191 & 0.16 \\
\hline & & $\mathrm{T} 2$ & 4.0 & 2.1 & 6.2 & .001 & 0.42 & 4.0 & 2.7 & 5.6 & $<.001$ & 0.45 & .724 & 0.04 \\
\hline & & T3 & 6.0 & 3.7 & 7.6 & .707 & 0.05 & 3.8 & 2.0 & 5.5 & .004 & 0.36 & $.014^{\dagger}$ & 0.31 \\
\hline \multirow{3}{*}{$\begin{array}{l}\text { average } \\
\text { pain } \\
\text { rating }\end{array}$} & {$[0-$} & $\mathrm{T} 1$ & 6.0 & 5.0 & 7.0 & & & 6.0 & 4.0 & 7.0 & & & .939 & 0.01 \\
\hline & & T2 & 5.0 & 4.0 & 6.0 & .008 & 0.36 & 5.0 & 4.0 & 6.0 & .002 & 0.41 & .488 & 0.09 \\
\hline & & T3 & 6.0 & 4.3 & 7.0 & .498 & 0.08 & 5.0 & 3.0 & 6.0 & .002 & 0.39 & $.044^{\dagger}$ & 0.25 \\
\hline \multicolumn{15}{|c|}{$r=0.1-0.29$ small effect $\mid r=0.30-0.49$ medium effect $\mid r \geq 0.50$ large effect } \\
\hline
\end{tabular}


Primary endpoints and follow-up group comparisons. At the three months follow-up (T3), HD-patients scored significantly lower on the painrelated disability scale compared to LD-patients (see Table 2 and Fig. 2A). In addition, HD-patients reported significantly lower average pain intensity compared to LD-patients at T3 (see Table 2 and Fig. 2B). The effect sizes of the between group comparisons can be considered as small (pain intensity) to medium (pain disability, see Table 2). Group differences clearly result from deteriorations between T2 and T3 in the LDgroup (Fig. 2). In detail, HD-patients reported significantly lower pain-related disability and average pain intensity ratings at T3 compared to T1 (medium effect size), while LD-patients showed no significant differences between T1 and T3.

Initial treatment effects between T1 and T2 and group comparisons. Both groups showed significant improvements between T1 and T2 in the pain disability scale and in average pain intensity, with medium effect sizes. The pain-related disability scale and average pain intensity ratings did not differ significantly between both groups at T2 (for details see Table 2).

\section{Discussion}

In this retrospective study, we aimed to compare the effects of different doses of IMPT (1 week, LD vs 4 weeks, HD) in matched pairs of patients with chronic pain and high pain-related disability prior to therapy at Jena University Hospital. Pain-related disability and average pain intensity showed significant and medium sized short-term improvements at the end of therapy (T1 vs. T2) in both groups. At the 3-month follow-up, improved outcomes of patients in the HD-group persisted and showed significantly lower pain-related disability and average pain intensity compared to T1 and, most remarkably, also compared to patients of the LD-group.

Baseline comparison and initial therapy effects. Based on the matching procedure, we found no significant differences in baseline data (e.g. duration of chronic pain) and pain scales at T1, indicating high comparability of both groups. Both patient groups showed significant improvements with medium-range effects in most of the pain scales directly after therapy. These values are similar to effect sizes reported by other interdisciplinary treatment programs with comparable durations and intensities ${ }^{33,34}$. In line with that, we found no significant differences between both groups at T2, indicating similar positive short-term effects.

Medium-term effects. Patients in the HD-group showed significantly lower pain-related disability and pain intensity compared to LD-patients at T3. The effect sizes of these differences were in the small to medium range. At T3, HD-patients showed significant improvements in painrelated disability and average pain ratings compared to T1. The effect sizes were in the range of the effect sizes reported by other German IMPT programs of comparable durations ${ }^{33,34}$. We assume that differences in medium-term effects between HD- and LD-patients mainly stem from differences in therapy duration because other therapeutic variables (e.g. therapeutic contents, involved disciplines, and contact hours per day) were highly comparable. In addition, both groups showed no baseline differences at T1 and the initial therapeutic effects of both therapy programs were similar. Most likely, a longer duration of IMPT ensures a longer-lasting transfer of functional pain management strategies in patients' daily life. Importantly, neither the pain-related disability scale nor the average pain intensity differed between T1 and T3 in LD-patients, indicating only short-lasting benefits and a return to baseline values after 3 months. In contrast, a recent study from Switzerland evaluated a 1week multidisciplinary treatment in an outpatient setting, revealing significant treatment effects after 3 and 12 months ${ }^{35}$. Nevertheless, these patients might not be comparable to our patient group because they suffered from unspecific back pain, had lower chronification grades, and received additional IMPT even after the intensive 1-week program, which may have played a crucial role in the maintenance of the therapy effects ${ }^{5}$.

We hypothesize that the LD-program seemingly was too short while the HD-program was long enough to maintain improvements up to 3 months in chronic pain patients with high pain-related disability, as usually present in inpatient or day-clinic IMPT programs. Nonetheless, the question of the optimal therapy duration remains unanswered ${ }^{5,20}$ and seems to be highly associated with patient characteristics, such as severity, disability and individual prognosis 22 .

In summary, after 3 months treatment effects in HD-patients were still significant, while LD-patients returned to T1 values. In addition, post-hoc analyses on all 114 patients treated in the HD-program with valid questionnaire data for all time points (T1 to T3 and T4: 6 months follow-up and T5: 12 months follow-up), revealed that pain-related disability was significantly reduced up to one year compared to T1 (see Supplement 4), indicating a stable long-term effect of the HD-program.

Strengths and limitations. The novelty and main strength and of the study is the high comparability of therapeutic features between therapy groups, e.g. interdisciplinary conceptual background, professionals involved, content and proportion of interventions. Therapy dose, i.e. mainly the total duration in therapy hours, is therefore assumed to be the most meaningful difference between both programs. Nonetheless, few limitations have to be considered. The small sample size, mono-centric setting and non-randomized patient allocation may have reduced validity and results should be interpreted with caution. Due to the retrospective data analyses the range of analyzed variables was rather small. A potential confounder might also be the differences of therapeutic setting, i.e. the inpatient setting of the LD-program compared to the day- 
clinic setting of the HD-program. Some studies showed better short-term ${ }^{36,37}$ and long-term ${ }^{37}$ improvements for inpatient compared to outpatient treatments. Nonetheless, our HD-program is rather intensive with daily interventions of approx. 6 hours per day and leave from work. In this respect, the basic difference between both therapy groups was rather not the therapy dosage or intensity, which can be concluded as high in both groups (between 4 and 6 hours per day), but the overnight stay and the amount of additional everyday activities beside therapy participation. In addition, the participation in the refresher days 3 months after therapy (2 days, approx. 10 hours) may also have sustained the positive outcomes for HD-patients.

Another confounding factor may be the difference in (group) sizes between both therapies with notably smaller group sizes in the LD-group. On one side, smaller group sizes may allow a higher level of individualized care. On the other side, some positive group effects may only come into effect in larger groups. HD-patients were treated some years later than LD-patients, however therapeutic content did not change in the meantime. Symptoms of depression and anxiety may have confounded the results of group comparisons but at least the number of patients with relevant symptoms of depression and anxiety according to cut-off scores (of the HADS (LD-patients) and DASS (HD-patients), respectively) did not differ significantly between groups at all time points.

Future studies on the effect of therapy dose should include multiple treatment centers using comparable therapy settings, comparable therapy contents and a standardized set of core outcome domains ${ }^{38}$. A randomized patient allocation would be preferable, although it might be associated with ethical concerns to deprive some patients with high symptom severity from therapies with appropriate doses.

Conclusion. Our study indicates that a high-dose IMPT is more effective in the medium term (3-months follow-up) than a low-dose therapy in chronic pain patients with high pain-related disability. Initial therapy effects were comparable, but improvements only persisted in the high-dose program. Despite methodological limitations the results are highly relevant to gain a deeper understanding of the association between therapy dose and effectiveness of IMPT.

\section{Declarations}

\section{Competing interests:}

The authors declare no competing interests.

\section{Date availability:}

The data that support the findings of this study are available from the corresponding author on reasonable request.

\section{Supplementary data:}

Supplementary data is provided for the online version of the article (attached to article).

\section{References}

1 Macfarlane, G. J. The epidemiology of chronic pain. Pain 157, 2158-2159, doi:10.1097/j.pain.0000000000000676 (2016).

2 Breivik, H., Collett, B., Ventafridda, V., Cohen, R. \& Gallacher, D. Survey of chronic pain in Europe: prevalence, impact on daily life, and treatment. European journal of pain 10, 287-333, doi:10.1016/j.ejpain.2005.06.009 (2006).

3 Gatchel, R. J., McGeary, D. D., McGeary, C. A. \& Lippe, B. Interdisciplinary chronic pain management: past, present, and future. The American psychologist 69, 119-130, doi:10.1037/a0035514 (2014).

4 Stanos, S. Focused review of interdisciplinary pain rehabilitation programs for chronic pain management. Current pain and headache reports 16, 147-152, doi:10.1007/s11916-012-0252-4 (2012).

5 Kamper, S. J. et al. Multidisciplinary biopsychosocial rehabilitation for chronic low back pain. Cochrane Db Syst Rev, doi:Artn Cd000963 10.1002/14651858.Cd000963.Pub3 (2014).

6 Borys, C., Lutz, J., Strauss, B. \& Altmann, U. Effectiveness of a Multimodal Therapy for Patients with Chronic Low Back Pain Regarding Pre-Admission Healthcare Utilization. PloS one 10, e0143139, doi:10.1371/journal.pone.0143139 (2015).

7 Preis, M. A. et al. Long-Term Outcomes of a Multimodal Day-Clinic Treatment for Chronic Pain under the Conditions of Routine Care. Pain research \& management 2018, 9472104, doi:10.1155/2018/9472104 (2018). 
8 Kaiser, U., Treede, R. D. \& Sabatowski, R. Multimodal pain therapy in chronic noncancer pain-gold standard or need for further clarification? Pain 158, 1853-1859, doi:10.1097/j.pain.0000000000000902 (2017).

9 Sabatowski, R. \& Kaiser, U. [Multimodal pain therapy: An established procedure?]. Schmerz 26, 644-646, doi:10.1007/s00482-012-1267-9 (2012).

10 Arnold, B. et al. Multimodal pain therapy for treatment of chronic pain syndrome. Consensus paper of the ad hoc commission on multimodal interdisciplinary pain management of the German Pain Society on treatment contents. Schmerz 28, 459-+, doi:10.1007/s00482-0141471-x (2014).

11 Nagel, B. \& Korb, J. [Interdisciplinary treatment. Long-lasting, effective, and cost-effective]. Der Orthopade 38, 907-908, 910-912, doi:10.1007/s00132-009-1482-y (2009).

12 Ringqvist, A., Dragioti, E., Bjork, M., Larsson, B. \& Gerdle, B. Moderate and Stable Pain Reductions as a Result of Interdisciplinary Pain Rehabilitation-A Cohort Study from the Swedish Quality Registry for Pain Rehabilitation (SQRP). Journal of clinical medicine 8, doi:10.3390/jcm8060905 (2019).

13 Casey, M. B., Smart, K. M., Segurado, R. \& Doody, C. Multidisciplinary-based Rehabilitation (MBR) Compared With Active Physical Interventions for Pain and Disability in Adults With Chronic Pain: A Systematic Review and Meta-analysis. Clin J Pain 36, 874-886, doi:10.1097/AJP.0000000000000871 (2020).

14 Guzman, J. et al. Multidisciplinary rehabilitation for chronic low back pain: systematic review. Brit Med J 322, 1511-1516, doi:DOI 10.1136/bmj.322.7301.1511 (2001).

15 van Geen, J. W., Edelaar, M. J. A., Janssen, M. \& van Eijk, J. T. M. The long-term effect of multidisciplinary back training - A systematic review. Spine 32, 249-255, doi:DOI 10.1097/01.brs.0000251745.00674.08 (2007).

16 IASP terminology, <https://www.iasp-pain.org/terminology?navltemNumber=576> (2019).

17 Frost, H., Lamb, S. E., Klaber Moffett, J. A., Fairbank, J. C. \& Moser, J. S. A fitness programme for patients with chronic low back pain: 2year follow-up of a randomised controlled trial. Pain 75, 273-279, doi:10.1016/s0304-3959(98)00005-0 (1998).

18 Mitchell, R. I. \& Carmen, G. M. The functional restoration approach to the treatment of chronic pain in patients with soft tissue and back injuries. Spine (Phila Pa 1976) 19, 633-642, doi:10.1097/00007632-199403001-00001 (1994).

19 Scascighini, L., Toma, V., Dober-Spielmann, S. \& Sprott, H. Multidisciplinary treatment for chronic pain: a systematic review of interventions and outcomes. Rheumatology 47, 670-678, doi:10.1093/rheumatology/ken021 (2008).

20 Waterschoot, F. P. C., Dijkstra, P. U., Geertzen, J. H. B., Reneman, M. F. \& Dijkstra, P. U. Dose or content? Effectiveness of pain rehabilitation programs for patients with chronic low back pain: A systematic review Reply. Pain 155, 1902-1903, doi:10.1016/j.pain.2014.05.017 (2014).

21 Dragioti, E., Bjork, M., Larsson, B. \& Gerdle, B. A Meta-Epidemiological Appraisal of the Effects of Interdisciplinary Multimodal Pain Therapy Dosing for Chronic Low Back Pain. Journal of clinical medicine 8, doi:10.3390/jcm8060871 (2019).

22 Haldorsen, E. M. H. et al. Is there a right treatment for a particular patient group? Comparison of ordinary treatment, light multidisciplinary treatment, and extensive multidisciplinary treatment for long-term sick-listed employees with musculoskeletal pain. Pain $\mathbf{9 5}$, 49-63 (2002).

23 Nagel, B., Gerbershagen, H. U., Lindena, G. \& Pfingsten, M. Entwicklung und empirische Überprüfung des Deutschen Schmerzfragebogens der DGSS. Schmerz 16, 262-270 (2002).

24 Klasen, B. W., Hallner, D., Schaub, C., Willburger, R. \& Hasenbring, M. Validation and reliability of the German version of the Chronic Pain Grade questionnaire in primary care back pain patients. Psychosoc Med 1, Doc07 (2004).

25 Vonkorff, M., Ormel, J., Keefe, F. J. \& Dworkin, S. F. Grading the Severity of Chronic Pain. Pain 50, 133-149, doi:Doi 10.1016/03043959(92)90154-4 (1992).

26 Zigmond, A. S. \& Snaith, R. P. The Hospital Anxiety and Depression Scale. Acta Psychiat Scand 67, 361-370, doi:D0I 10.1111/j.16000447.1983.tb09716.x (1983). 

1995).

28 Lovibond, P. F. \& Lovibond, S. H. The Structure of Negative Emotional States - Comparison of the Depression Anxiety Stress Scales (Dass) with the Beck Depression and Anxiety Inventories. Behav Res Ther 33, 335-343, doi:Doi 10.1016/0005-7967(94)00075-U (1995).

29 Nilges, P. \& Essau, C. Depression, anxiety and stress scales. DASS-A screening procedure not only for pain patients. Schmerz 29, 649-657, doi:10.1007/s00482-015-0019-z (2015).

30 Ho, D. E., Imai, K., King, G. \& Stuart, E. A. Matchlt: Nonparametric Preprocessing for Parametric Causal Inference. J Stat Softw 42 (2011).

31 R: A language and environment for statistical computing. (R Foundation for Statistical Computing, Vienna, Austria, 2014).

32 Fritz, C. O., Morris, P. E. \& Richler, J. J. Effect Size Estimates: Current Use, Calculations, and Interpretation. J Exp Psychol Gen 141, 2-18, doi:10.1037/a0024338 (2012).

33 Pohlmann, K., Tonhauser, T., Joraschky, P. \& Arnold, B. The Dachau multidisciplinary treatment program for chronic pain. Efficacy data of a diagnosis-independent multidisciplinary treatment program for back pain and other types of chronic pain. Schmerz 23, 40-46, doi:10.1007/s00482-008-0727-8 (2009).

34 Schutze, A. et al. Evaluation of a multimodal pain therapy at the University Pain Centre Dresden. Schmerz 23, 609-+, doi:10.1007/s00482-009-0827-0 (2009).

35 Reck, T., Dumat, W., Krebs, J. \& Ljutow, A. [Outpatient multimodal pain therapy : Results of a 1-week intensive outpatient multimodal group program for patients with chronic unspecific back pain - retrospective evaluation after 3 and 12 months]. Schmerz, doi:10.1007/s00482017-0211-4 (2017).

36 Harkapaa, K., Jarvikoski, A., Mellin, G. \& Hurri, H. A Controlled-Study on the Outcome of Inpatient and Outpatient Treatment of Low-Back Pain .1. Pain, Disability, Compliance, and Reported Treatment Benefits 3 Months after Treatment. Scand J Rehabil Med 21, 81-89 (1989).

37 Williams, A. C. D. et al. Inpatient vs outpatient pain management: Results of a randomised controlled trial. Pain 66, 13-22, doi:Doi 10.1016/0304-3959(96)02996-X (1996).

38 Kaiser, U. et al. Developing a core outcome domain set to assessing effectiveness of interdisciplinary multimodal pain therapy: the VAPAIN consensus statement on core outcome domains. Pain 159, 673-683, doi:10.1097/j.pain.0000000000001129 (2018).

39 Hinz, A. \& Brahler, E. Normative values for the Hospital Anxiety and Depression Scale (HADS) in the general German population. $J$ Psychosom Res 71, 74-78, doi:10.1016/j.jpsychores.2011.01.005 (2011).

40 Pfingsten, M. et al. www.dggs.org/deutscher-schmerzfragbogen/, 2015).

\section{Figures}




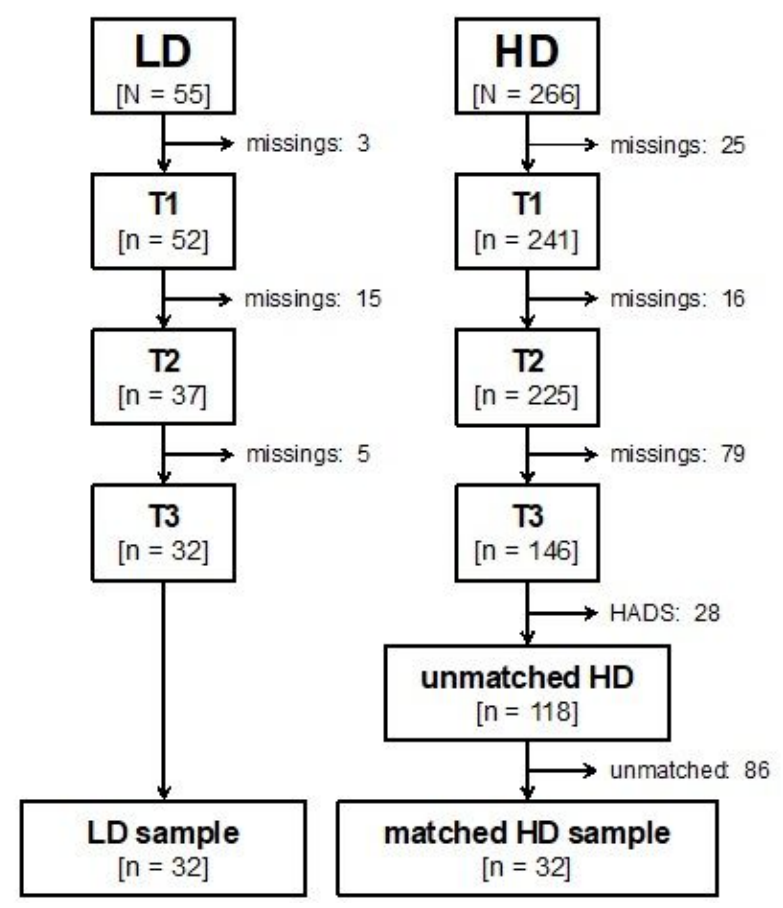

Figure 1

STROBE diagram for patients in the low-dose (LD) and high-dose (HD) therapy program. Number of patients with incomplete data-sets at the beginning (T1), end of the therapy (T2), and follow-up (T3) are displayed.

A

pain disability scale

$\pitchfork$ LD $\bullet$ HD

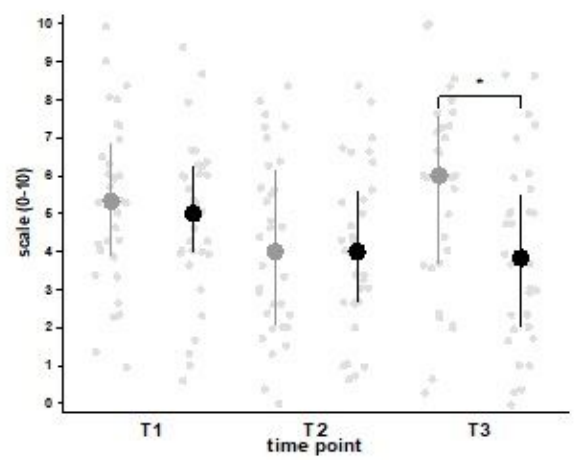

B
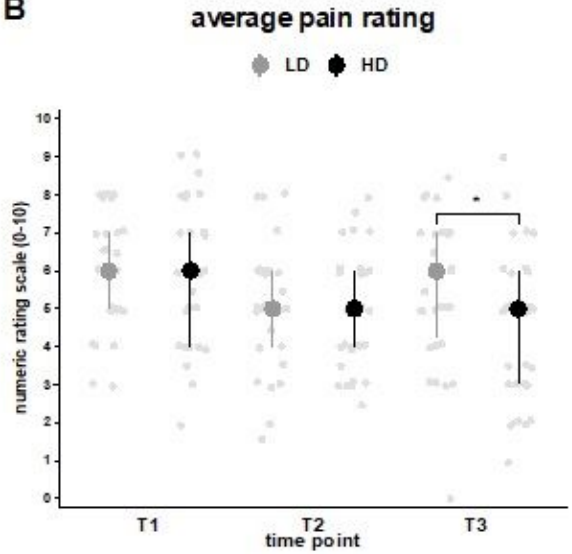

Figure 2

Pain-related disability scale (A) and average pain intensity (B) for the low dose (LD) and high dose (HD) therapy group at the beginning (T1), end of the therapy (T2), and follow-up (T3). The dots indicate group medians and error bars indicate the 1st and 3rd quartile. Significant differences between groups are marked with stars.

\section{Supplementary Files}

This is a list of supplementary files associated with this preprint. Click to download.

- SupplementIMSTDoseScRepfinal.docx 